\title{
Commentary on 'a Reflexive Account of Reusing Qualitative Data: Beyond Primary/secondary Dualism' (Libby Bishop)
}

\author{
by Mildred Blaxter \\ Sociological Research Online, Volume 12, Issue 3, \\ $<$ http://umw. socresonline.org.uk/12/3/7.html> \\ doi:10.5153/sro. 1561
}

Received: 22 May 2007 Accepted: 22 May 2007 Published: 30 May 2007

\section{Introduction}

1.1 This paper is an interesting contribution to the currently lively topic of the secondary analysis of qualitative data. As an engaging, accessible, and honest account of (in her own phrase) "how your mind works" during the process of reusing data from different historical periods it is most welcome: the endeavour is both difficult and courageous.

1.2 Researchers commonly place a study within their intellectual history, but practical detail like this is quite rare. It is as necessary, she suggests, to be reflexive about an encounter with secondary materials as it is about the actual encounter with respondents in real time. She explains clearly why this topic and method were chosen, in personal and practical terms - that is, notably the need to obtain first-hand experience of the use of Qualidata data sets, to better advise both depositors and users. Any researcher will emphathise with her detailed accounts of her approaches to her material, and the phases of her research - the "meandering but not atypical path" to reassess what data were relevant. She concludes, convincingly, that the processes of doing primary and secondary research are more alike than not.

1.3 There is general agreement that qualitative data, as the product of the reflexive relationship between researchers and researched, may produce problems for the reuse of data by those not involved in its production (Hammersley 1997). There is a realist view of secondary analysis, as Bishop notes, that data are "out there" and can be regarded as simply "given". However, she not only emphasizes being transparent about her own method, but also dwells on how the original studies were conducted and the nature of the interactions displayed in the data transcripts. Despite her comment that "Data are recontextualised and coconstructed whether reading transcripts or doing and interview in real time", clearly she would support the more common view, that attempting to understand fieldwork relationships and the context of the original data are among the special features - and difficulties - of qualitative secondary analysis.

1.4 But the "proof of the pudding..." test remains. The crucial issue, in the end, is whether a secondary analysis does offer a new perspective or useful results. Remembering that the two analyses reported here are only part of Bishop's larger research programme, and the paper is an account of methodology rather than actual findings, the theme is promising. What can historical data, exploring beliefs and practices from which current uses of convenience foods have emerged, tell us about domestic food consumption as a setting in which the discourse of choice - described as the "ubiquitous mantra of neoliberalism" - is enacted?

1.5 This commentary will focus on only one of the two research projects used, .the "Mothers and Daughters" study of Blaxter and Paterson (1982). There is, of course, a difficulty in that we do not in fact have the substantive results of the secondary analysis, so it is not really possible to assess its outcome. However, some clues can be found in this account. It seems that three problems emerge: problems relating to the research context, those associated with the particular historical and social context of the original work, and issues about possibly divergent purposes of the primary and secondary studies.

\section{Institutional and practical context of the research}

2.1 Bishop acknowledges the importance of paying particular attention to the actual conduct of the original study, and the nature of the relationship between researcher and researched. Because, except in a methods paper, the standard journals would simply not have room or inclination for much navel-gazing, this material can rarely be found in published papers. The data required are probably in research reports, if anywhere, or in research diaries. Hinds et al (1997) suggest that "Sensitivity of secondary researchers to the context of the primary study is crucial", and if this material is not archived, then "access to it and to members of the study team, if available, could and should be negotiated". Certainly, secondary analysists must resist second-guessing things about the conduct of the study that they cannot possibly know. Misunderstandings can of course be corrected, if the primary researchers are available to comment, but 
even if this is done traces of assumption about "this is how it was" tend to embed themselves in the analysis and remain.

2.2 The analysis here picked up hints about the conduct of the study (eg some personal details about the researchers) but not others, at the situational and organizational levels, which are arguably more important. These relate, for instance, to the collegiate organisation of the ESRC's "transmitted deprivation" programme, and to the institutional setting in which the research was conducted, including the interest of some of the original medical researchers who first studied these respondents in a different way and on different topics in 1950. Many things influenced the direction and nature of the data-collection. There is a sort of archeological task here, with layers of information to be sifted from all the papers and reports from a project and from others associated with it. This is particularly true if the secondary analysis relates to data which were only part of a larger study.

\section{Social contexts}

3.1 Hammersley (1997) also commented, " To understand behaviour one must learn the culture which informs it", and a more serious potential problem of secondary analysis relates to what might be a neglect of, or even a misunderstanding of, the social context, local culture, and history of the research setting and its population. Bishop notes that not all researchers necessarily share the culture of their subjects, and how one attempts, as a "foreigner", to build up sufficient rapport is a common topic of discussion in qualitative work. Primary researchers would not, however, devote perhaps three years of their lives to attempting to understand a particular group without a great deal of background study as well as first hand observation. This background knowledge informs their own analyses but may not be made explicit in every paper from the study.

3.2 Particular problems may arise where data sets belong to different historical periods during which the phenomenon of interest may have taken on different forms. The term "convenience food" was certainly unknown to the population studied in the early 1980s. Frozen food was not used and was viewed with some suspicion. "Convenient" foods such as custard powder or packeted products, and some tins, were much used, but regarded as cheap and traditional cooking ingredients. One of Bishop's research questions was "Under what conditions was convenience food accepted?", but this begs the question of what is to be counted as a convenience food, as she notes, as well as what food was in fact available in this area at this time.

3.3 It is clear that criteria for "good" or "bad" food are similarly historically situated. Without further information (difficult to discuss in the one original paper to which the secondary analysis refers, though it was to some extent in the book of the study and in other papers which derived from it) it would be impossible to know what this history was. though it would have been clearer to contemporary readers. This was a group of women with predominantly rural childhoods in an area of Scotland during a period of particular economic depression. Home-grown and home-cooked food was an economic necessity, but it was also bound up with the mythical "golden days of childhood" and with the strong moral climate of selfdenial and restraint.

3.4 There are some hints in Bishop's paper of the dangers of attempting to apply understandings of one period to the culture of another For instance, she notes that "tinned food as protection from want was surely more prominent for the grandmothers than would be typical today": no, much tinned food, far from being "protection from want", represented a degree of (unnecessary) luxury or indulgence. As the original paper "The goodness is out of it" said, these women had grown up during war years, when those things which were severely rationed were seen as necessities, and those which had been unavailable were indulgences. They belonged to the generation who, as the novelist Margaret Drabble has noted in her book The Ice Age, "had always been astonished at their own purchasing power whenever they bought a pound of bananas or a small pot of double cream". Their common social experience - a particular national culture with regard to food, childhood during economic depression, growing up during a war, and the perception of rapid social change since - was very obvious in their attitudes.

3.5 Of course, as Bishop notes, interviews about values and attitudes must always be considered as presentations, framed in perceptions of perceived acceptability. Again, however, any speculation about the possibility of the suppression of anti-health practices and attitudes must be informed by knowledge of the particular public discourse current at this time and in this culture.

\section{Purposes of the studies}

4.1 The literature on secondary analysis agrees that a significant problem arises when the research question asked in the secondary analysis is different from the original research question. Indeed, this is always likely to be the case, since even if the research climate did not change historically, there is little 
attraction in simple repetition. How far can the research purpose differ without invalidating the effort? (Hinds et al 1997). The substantive intention of Bishop's use of these data - to examine attitudes and practices about early forms of processed foods and about food choices at meals - was not, as she makes clear. a particular focus of the original study. There. food, and specifically the feeding of infants and children emerged as one topic of interest within the overall theme of intergenerational attitudes to the health and care of children in the early 1980s. The interviewers were aware of many other potential threads about food which might have been followed up, but were not, because they were not relevant to the study's purpose.

4.2 Savage (2007) noted that the data archived in qualitative studies may contain considerably more material than was reported in published papers, but it requires investment to discover exactly what this consists of and bring its potential for re-use to light. In the study being used here, there was observational data about meals which was not reported. though some information might be derived from various other papers from the study. Is the "data" which the secondary analysis is based complete, if it relies solely on what is in the transcripts? Or is it partial, and selected on grounds which apply to the primary research question but not that of the secondary analysis? "Making things visible means making other things invisible" (Fielding and Fielding, 2000), and out of the enormous amount which any qualitative study collects, only that which is defined as data in relation to the original research question is formally recorded.

4.3 An example concerns the observed context within which food choices were made, and the informal discussions with husbands and other family members about a meal in preparation, which took place in the interviewer's presence (and to which the interviewer was sometimes invited by these ever-generous respondents), not transcribed as part of the formal interview. A vignette: there was a young mother of a sixyear old boy and a baby of a few months, living in a tenement block with no local shops within easy walking distance, and forced to rely for food purchases on the daily travelling vans which toured the estate. She lived on the edge of "managing", lurching from day to day with catering and budgeting. One interview was interrupted by the chimes of the travelling grocery, and the tape was turned off while she looked for her purse, and explained that the six-year old was a "good little shopper" on whom she had to rely, since she did not want either to wake the baby up to carry him down several flights of stairs, nor leave him alone in the flat. "Get what you like for tea [evening meal]", she said, and twenty minutes later her son returned triumphant with a tin of strawberries, evaporated milk and a bag of buns.

4.4 This specific example relates to the younger generation of respondents to the original study, whose attitudes to "luxuries" had changed. Their interviews were not deposited in the Archive and so not available for secondary research. In general, however, what may be missing from Bishop's account is the observed way in which practical constraints (including often what corner shops or travelling vans choose to stock) determined food choice for those of both generations who lived in large estates or tenement blocks and struggled with their housekeeping.

4.5 The purpose of the original study was the comparison of attitudes within the two generations. Sadly, consideration of continuity and change might have served the purposes of the secondary analysis very well. The use of this was precluded, however, by the definition of the data to be analysed as solely the deposited "raw" data, excluding other analyses or research reports.

\section{Conclusion}

5.1 Despite its illustration of these problems. Bishop's paper remains an interesting contribution to the principles and practice of secondary analysis. A final thought, however, relates to one potential use of secondary analysis which is consistent with an interpretive qualitative methodology - that is, to understand the research process itself, unravelling, for instance, the way in which the processes of abstraction which are inherent in any research are never neutral, but part of the constructions and omissions which inevitably take place in the social science canon, as Savage (2007) demonstrated with reanalyses of Bott's "Family and Social Network" and Goldthorpe and Lockwood's "Affluent Worker" collection. There are clear historical influences in both the primary and secondary analyses here. Choices made by both authors and publishers about the place and form of the publications which these analyses give rise to are also part of this construction of knowledge. In this light both the primary and secondary analyses here become part of a new analysis. But perhaps this takes reflexivity too far!

\section{References}

HAMMERSLEY, M. (1997) 'Qualitative data archiving: some reflections on its prospects and principles, Sociology, Vol 31, pp.131-41.

HINDS, P.S, Vogel R.J., and Clarke-Steffen L. (1997) 'The possibilities and pitfalls of doing a secondary analysis of a qualitative data ser', Qualitative Health Research, Vol. 7, pp 415-23. 
FIELDING, N.J and Fielding, J.L. (2000) 'Resistance and adaptation to criminal identity: using secondary anausis to evaluate class studies of crime and devience' Sociology, Vol. 34, pp 671-89.

SAVAGE. M. (2007) ‘Revisiting class qualitative studies', Forum: Qualitative Social Research, Vol. 6, No. I, Art. 3l. 